\title{
Exosomal miR-17-3p Alleviates Programmed Necrosis in Cardiac Ischemia/Reperfusion Injury by Regulating TIMP3 Expression
}

\author{
Zhuyuan Liu (D, Didi Zhu, Fuchao Yu, Mingming Yang, Dan Huang, Zhenjun Ji, Wenbin Lu, \\ and Genshan Ma
}

Department of Cardiology, Zhongda Hospital, Southeast University, Hunan Road, Nanjing, 210009 Jiangsu, China

Correspondence should be addressed to Genshan Ma; magenshan@hotmail.com

Received 4 September 2021; Revised 17 December 2021; Accepted 6 January 2022; Published 25 January 2022

Academic Editor: Ding-Sheng Jiang

Copyright (c) 2022 Zhuyuan Liu et al. This is an open access article distributed under the Creative Commons Attribution License, which permits unrestricted use, distribution, and reproduction in any medium, provided the original work is properly cited.

\begin{abstract}
Objective. Myocardial ischemia/reperfusion (I/R) injury can aggravate myocardial injury. Programmed necrosis plays a crucial role in this injury. However, the role of exosomal miRNAs in myocardial I/R injury remains unclear. Therefore, this study is aimed at exploring the function and mechanism of exosomal miR-17-3p in myocardial I/R injury. Methods. The myocardial I/R injury animal model was established in C57BL/6 mice. Exosomes were identified using transmission electron microscopy (TEM), nanoparticle tracking analysis (NTA), and Western blotting. Programmed necrosis was detected by PI staining. Heart function and myocardial infarct size were evaluated using echocardiography and triphenyl tetrazolium chloride (TTC) staining, respectively. Histopathological changes were visualized by hematoxylin and eosin ( $\mathrm{H} \& \mathrm{E})$ and Masson staining. The regulation of TIMP3 expression by miR-17-3p was verified using a dual-luciferase reporter assay. Lactate dehydrogenase (LDH) and tumor necrosis factor- $\alpha$ (TNF- $\alpha$ ) levels were measured by enzyme-linked immunosorbent assays (ELISA). TIMP3 expression was measured by quantitative reverse transcription-polymerase chain reaction (qRT-PCR) and Western blotting. Results. We demonstrated that miR-17-3p was significantly downregulated in peripheral blood exosomes after cardiac I/R injury. Further analysis indicated that exosomal miR-17-3p attenuated $\mathrm{H}_{2} \mathrm{O}_{2}$-induced programmed necrosis in cardiomyocytes in vitro. Moreover, TIMP3 was a target for miR-17-3p. TIMP3 affected $\mathrm{H}_{2} \mathrm{O}_{2}$-induced programmed necrosis in cardiomyocytes. This effect was modulated by miR-17-3p in vitro. Furthermore, exosomal miR-17-3p greatly alleviated cardiac I/R injury in vivo. Conclusions. The present study demonstrated that exosomal miR-17-3p alleviated the programmed necrosis associated with cardiac I/R injury by regulating TIMP3 expression. These findings could represent a potential treatment for I/R injury.
\end{abstract}

\section{Introduction}

Myocardial ischemia/reperfusion (I/R) injury is a common pathophysiological phenomenon in the clinic [1]. Reperfusion is an effective treatment for ischemic heart disease, such as acute myocardial infarction (AMI). However, reperfusion is often performed after the blood flow in myocardial tissue is interrupted for a certain period of time and then reestablished. Under those conditions, reperfusion fails to restore normal heart function and aggravates its dysfunction and structural damage, leading to irreversible damage that could affect the prognosis of patients with myocardial infarction $[2,3]$. Therefore, clarifying the mechanism of myocardial $\mathrm{I} / \mathrm{R}$ injury has important clinical significance for reducing or eliminating this injury.
The pathogenesis of myocardial I/R injury is complex, and oxygen free radicals, calcium overload, and inflammatory mediators are recognized mechanisms $[1,4]$. MicroRNAs (miRNAs) are small noncoding RNAs with a length of about 22 nucleotides that are widely present in various organisms and regulate growth, development, and apoptosis. miRNAs (e.g., microRNA-374a, miR-204-3p, and microRNA-193b) are involved in various diseases and myocardial I/R injury [5-7]. In addition, an increasing number of studies have found that exosomes and exosomal miRNA play a crucial role in myocardial I/R injury. For example, mesenchymal stem cell-derived exosomal miRNA-181a and miR-182 attenuate myocardial $\mathrm{I} / \mathrm{R}$ injury by influencing the inflammatory response and macrophage polarization, respectively $[8,9]$. Exosomes derived from the coronary serum of patients with 
myocardial infarction promote angiogenesis through the miRNA-143/IGF-IR pathway [10].

Programmed cell necrosis is a new type of cell death that is different from apoptosis and traditional necrosis [11]. An increasing number of studies have demonstrated that programmed necrosis plays an important role in myocardial I/ $\mathrm{R}$ injury [12]. Several studies also found that miRNA can protect against myocardial I/R injury by regulating this process. For example, miRNA-325-3p protects the heart after myocardial infarction by inhibiting RIPK3 and programmed necrosis in mice [13]. In addition, miRNA-103 and miRNA107 regulate programmed necrosis and myocardial I/R injury by targeting FADD [14]. miR-17-3p contributes to exercise-induced cardiac growth and protects against myocardial I/R injury [15]. miR-17-3p plays a crucial role in different diseases by regulating various kinds of biological functions. Rosuvastatin attenuates myocardial I/R injury by mediating autophagy by upregulating miR-17-3p [16]. Recently, Yuan et al. demonstrated that $H / R$ injury in H9C2 cardiomyocytes is reduced by modulating inflammatory signaling regulated by $\mathrm{miR}-17-3 \mathrm{p}$ [17]. Moreover, we found that increasing miR-17-3p levels from serum extracellular vesicles promotes H9C2 proliferation [18]. However, the function and mechanism of exosomal miR-17-3p in programmed necrosis during myocardial $\mathrm{I} / \mathrm{R}$ injury remain unclear. This study is aimed at exploring the mechanism of exosomal miR-17-3p in programmed necrosis during myocardial I/R injury in vitro and in vivo, providing a potential therapeutic target for cardiac I/R injury.

\section{Materials and Methods}

2.1. Exosome Isolation and Characterization. Exosomes were isolated using the Total Exosome Isolation Kit (4484450, Invitrogen, USA) according to the manufacturer's instructions. The size distribution and concentration of exosomes were measured by nanoparticle tracking analysis (NTA) using a ZetaView instrument (Particle Metrix). For transmission electron microscopy (TEM), exosomes were sedimented by ultracentrifugation (Beckman TL-100) at 200,000 $\times \mathrm{g}$ for $20 \mathrm{~h}$ through a sucrose gradient and fixed in 3\% $(\mathrm{w} / \mathrm{v})$ glutaraldehyde and $2 \%$ paraformaldehyde in cacodylate buffer ( $\mathrm{pH}$ 7.3). The fixed exosomes were applied to a continuous carbon grid and negatively stained with $2 \%$ uranyl acetate. The samples were examined with a Philips CM10 electron microscope. The exosomal markers (CD63, CD9, and TSG101) were also detected by Western blot.

2.2. Cell Culture, Transfection, and $\mathrm{H}_{2} \mathrm{O}_{2}$ Treatment. Rat primary cardiomyocytes were isolated and cultured in Dulbecco's modified Eagle's medium (Gibco, Thermo Fisher Scientific Inc.) containing $10 \%$ fetal bovine serum (Gibco, USA) at $37^{\circ} \mathrm{C}$ in an incubator with $95 \%$ air and $5 \% \mathrm{CO}_{2}$. Cells were used in experiments at $70 \%$ confluency. $\mathrm{H}_{2} \mathrm{O}_{2}$ treatment to cause the ischemic reperfusion (I/R) injury was performed as previously described $[14,19,20]$. Briefly, the cardiomyocytes were treated with $500 \mu \mathrm{M} \mathrm{H}_{2} \mathrm{O}_{2}$ (Sigma, USA), $10 \mu \mathrm{g} / \mathrm{mL}$ exosomes, or both $500 \mu \mathrm{M} \mathrm{H}_{2} \mathrm{O}_{2}$ and $10 \mu \mathrm{g} /$ $\mathrm{mL}$ exosomes for $24 \mathrm{~h}$. For transfection, the cardiomyocytes were transfected using Lipofectamine ${ }^{\circledR} 3000$ reagent (Invitrogen; Thermo Fisher Scientific Inc.), according to the manufacturer's protocol. The cells were harvested $48 \mathrm{~h}$ after transfection for subsequent experiments. The sequences of the miR-17-3p inhibitor, small interfering RNAs (siRNAs) targeting TIMP3, and the negative control siRNAs (si-NC) were designed and synthesized by KeyGEN BioTECH. The sequences were as follows: miR-17-3p inhibitor $5^{\prime}$-UUCU CCGAACGUGUCACGUTT-3; Si TIMP3 sense, $5^{\prime}$-GGUC UACACUAUUAAGCAAAU-3'; Si TIMP3 antisense, 5' UUGCUUAAUAGUGUAGACCAG-3.

2.3. Cell Necrosis Assays. Cell necrosis was detected by PI staining. Briefly, cardiomyocytes were washed three times with PBS after $\mathrm{H}_{2} \mathrm{O}_{2}$ treatment. The cells were stained with $1.5 \mu \mathrm{M}$ PI on ice for $5 \mathrm{~min}$ and then fixed with $4 \%$ PFA on ice for $30 \mathrm{~min}$ after washing with PBS three times. The cardiomyocytes were counterstained with DAPI and examined with a Nikon Eclipse Ti-S fluorescence microscope. Image analysis was performed with Image-Pro Plus software. The percentage of necrotic cell death was calculated by the total number of PI-positive nuclei/total DAPI-stained nuclei.

\subsection{Quantitative Reverse Transcription-PCR (qRT-PCR).} Total RNA was extracted from the cardiomyocytes and tissues using TRIzol ${ }^{\circledR}$ reagent (Biosharp BS259A). The quality and concentration of the RNA were measured using a NanoDrop, and $1 \mu \mathrm{g}$ RNA was reverse transcribed into cDNA using the PrimeScript RT Reagent Kit with gDNA Eraser (Vazyme MR101-01/02) for mRNA and Super RT Kit (Bioteke Biotechnology Co. Ltd.) for miRNA, according to the manufacturers' protocols. qRT-PCR was performed using SYBR Premix Ex Taq II (Vazyme Q711-02) and the manufacturer's protocol. cDNA was amplified using the following thermocycling conditions: $94^{\circ} \mathrm{C}$ for $10 \mathrm{~min}\left(94^{\circ} \mathrm{C}\right.$ for $20 \mathrm{~s}, 60^{\circ} \mathrm{C}$ for $20 \mathrm{~s}$, and $72^{\circ} \mathrm{C}$ for $20 \mathrm{~s}$ ) for 40 cycles; relative gene expression was analyzed using the $2^{-\Delta \Delta C t}$ method. $\beta$-Actin and U6 were used as the internal standards for mRNA and miRNA, respectively. The primers were synthesized by Tsingke Biotechnology Co. Ltd., and the primer sequences are listed in Table 1.

2.5. Western Blot Analysis. Total protein was extracted from cultured cells or tissues using RIPA buffer (Thermo Fisher Scientific). Protein concentrations were determined using the BCA assay (Biosharp BL521A). Equal amounts of protein $(20 \mu \mathrm{g})$ were separated on SDS-PAGE gels and transferred to polyvinylidene difluoride (PVDF) membranes (Millipore). The membranes were blocked with 5\% nonfat milk in 10\% Tris-buffered saline Tween 20 (TBST) at room temperature for $1 \mathrm{~h}$. The membranes were then incubated with CD9 (1:2000, ab92726, Abcam), CD63 (1:2000, ab134045, Abcam), TSG101 (1:2000, ab125011, Abcam), TIMP3 (1:1000, ab276134, Abcam), ZNF28 (1:1000, PA548229, Invitrogen), FAM83F (1:1000, ab272651, Abcam), and Neurog1 (1:500, sc-100332, Santa Cruz) primary antibodies overnight at $4^{\circ} \mathrm{C}$. The membranes were incubated with goat anti-rabbit peroxidase-conjugated secondary antibodies (Biosharp BL003A) at room temperature for $2 \mathrm{~h}$. The signal was detected using the ECL system (Millipore, 
TABLE 1: qRT-PCR primers.

\begin{tabular}{|c|c|}
\hline Gene & Sequence $\left(5^{\prime}-3^{\prime}\right)$ \\
\hline U6-RT-F & CTCGCTTCGGCAGCACA \\
\hline U6-RT-R & AACGCTTCACGAATTTGCGT \\
\hline URP & CTCAACTGGTGTCGTGGA \\
\hline hsa-miR-17-3p mature sequence & ACUGCAGUGAAGGCACUUGUAG \\
\hline hsa-miR-17-3p reverse primer & CTCAACTGGTGTCGTGGAGTCGGCAATTCAGTTGAGCTACAAGT \\
\hline rno-miR-17-1-3p mature sequence & ACUGCAGUGAAGGCACUUGUGG \\
\hline rno-miR-17-3p reverse primer & CTCAACTGGTGTCGTGGAGTCGGCAATTCAGTTGAGCCACAAGT \\
\hline miR-17-3p-F & ATAGACUGCAGUGAAGGC \\
\hline a-SMA-mouse-F & CCCAACTGGGACCACATGG \\
\hline a-SMA-mouse-R & TACATGCGGGGGACATTGAAG \\
\hline Col3a1-mouse-F & CTGTAACATGGAAACTGGGGAAA \\
\hline Col3a1-mouse-R & CCATAGCTGAACTGAAAACCACC \\
\hline Colla1-mouse-F & CTGGCGGTTCAGGTCCAAT \\
\hline Colla1-mouse- $\mathrm{R}$ & TTCCAGGCAATCCACGAGC \\
\hline ANP-mouse-F & GCTTCCAGGCCATATTGGAG \\
\hline ANP-mouse-R & GGGGGCATGACCTCATCTT \\
\hline BNP-mouse-F & AGTCCTTCGGTCTCAAGGCA \\
\hline BNP-mouse-R & CCGATCCGGTCTATCTTGTGC \\
\hline$\alpha$-mhc-mouse-F & TGAGTGGGAGTTTATCGACTTCG \\
\hline$\alpha$-mhc-mouse- $\mathrm{R}$ & CCTTGACATTGCGAGGCTTC \\
\hline$\beta$-mhc-mouse-F & CCTGCGGAAGTCTGAGAAGG \\
\hline$\beta$-mhc-mouse-R & CTCGGGACACGATCTTGGC \\
\hline Actin-mouse-F & GTGACGTTGACATCCGTAAAGA \\
\hline Actin-mouse- $\mathrm{R}$ & GCCGGACTCATCGTACTCC \\
\hline
\end{tabular}

Massachusetts, USA), according to the manufacturer's instructions. The relative protein expression was analyzed using Image software and presented as the density ratio between the target protein and the $\beta$-actin internal control.

2.6. Bioinformatics Analysis and Dual-Luciferase Reporter Assay. TargetScan (http://www.targetscan.org/vert_72/) was used to identify the potential miR-17-3p binding sequences in TIMP3. Wild-type and mutant $3^{\prime}$ UTR of TIMP3 sequences were cloned into the pmirGLO vector downstream of the luciferase reporter gene. The reporter constructs were cotransfected into cardiomyocytes with a miR-17-3p mimic or mimic NC using Lipofectamine 3000 according to the manufacturer's instructions. Luciferase activity was measured $48 \mathrm{~h}$ after transfection using the Dual-Luciferase Reporter Assay System (Promega, Madison, WI) following the manufacturer's instructions.

2.7. Myocardial I/R Model in C57BL6 Mice. Ten-week-old C57BL/6 mice were purchased from the Comparative Medicine Center of Yangzhou University and housed in a specific-pathogen-free facility with a 12 light-dark cycle under $21 \pm 2{ }^{\circ} \mathrm{C}$ with $55 \%$ air humidity. The myocardial I/R model was established as previously described [20]. Briefly, mice were anesthetized with $1.5-2.0 \%$ isoflurane and ventilated with room air using a rodent ventilator. A thoracotomy was performed at the fourth intercostal space to expose the heart and left anterior descending coronary artery (LAD). For the negative control, the LAD was not ligated. For functional studies, C57BL/6 mice were intravenously injected with $5 \mathrm{mg} / \mathrm{mL}$ exosomes $(150 \mu \mathrm{L})$ or the $100 \mathrm{nM} \mathrm{miR}-17$ $3 \mathrm{p}$ inhibitor $(100 \mu \mathrm{L})$ one day before myocardial I/R injury. All animals were anesthetized by isoflurane inhalation $(1.5-2 \%)$ and then euthanized by cervical dislocation. The myocardial infarct size was detected with Evans blue dye and 2,3,5-triphenyl-2H-tetrazolium chloride (TTC) staining. The area of infarction and total area of the transverse section were measured using NIH ImageJ software. The infarct size $(\%)=$ infarct size/total area of transverse section $\times 100 \%$. Echocardiography was performed on the rats in each group using a MyLab $30 \mathrm{CV}$ ultrasound system (Esaote, S.P.A, Genoa, Italy) with $10 \mathrm{MHz}$ linear ultrasonic transducers. The ejection fraction (EF (\%)), fraction shortening (FS $(\%)$ ), and heart rate were measured. The left ventricular end-diastolic diameter (LVEDd) and left ventricular endsystolic diameter (LVEDs) were also measured. LVEF (\%)= [(left ventricular end-diastolic volume-left ventricular endsystolic volume)/left ventricular end-diastolic volume] $\times 100 \%$. Lactate dehydrogenase (LDH) and TNF- $\alpha$ were determined using the $\mathrm{LDH}$ assay kit (Nanjing Jiancheng Bioengineering Institute A020-2-2) and TNF- $\alpha$ enzyme-linked immunosorbent assay (Elabscience E-EL-M0049C), according to the manufacturers' protocols. Absorbance was detected at a wavelength of $450 \mathrm{~nm}$ using an ultramicro microporous plate 

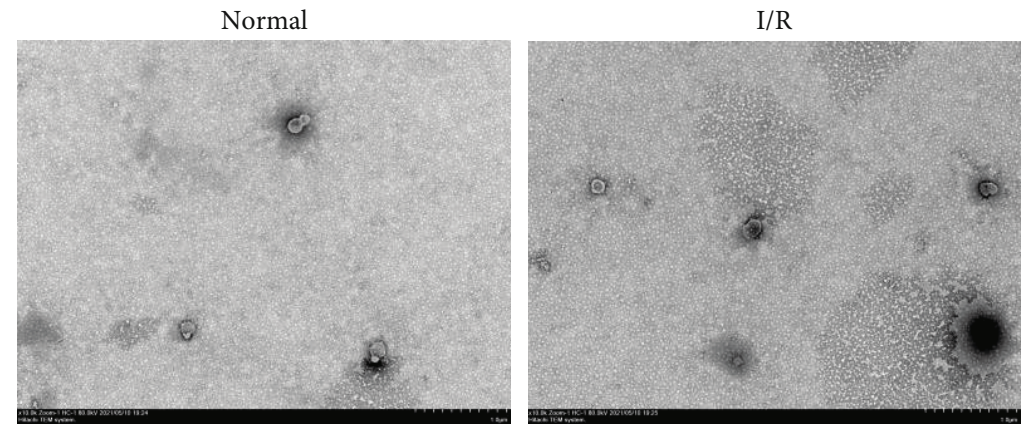

(a)

Normal

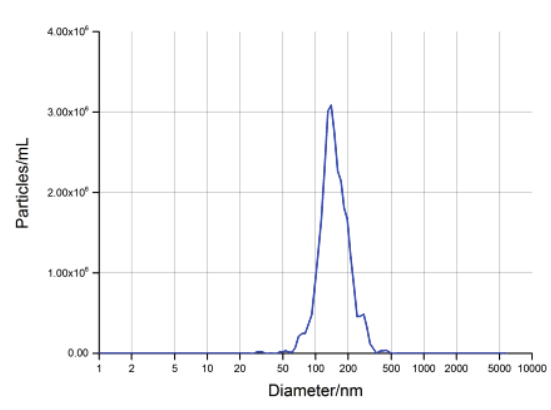

(b)

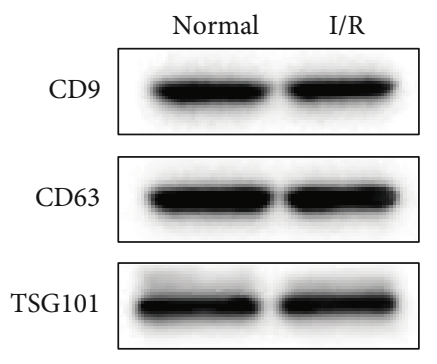

(c)
$\mathrm{I} / \mathrm{R}$
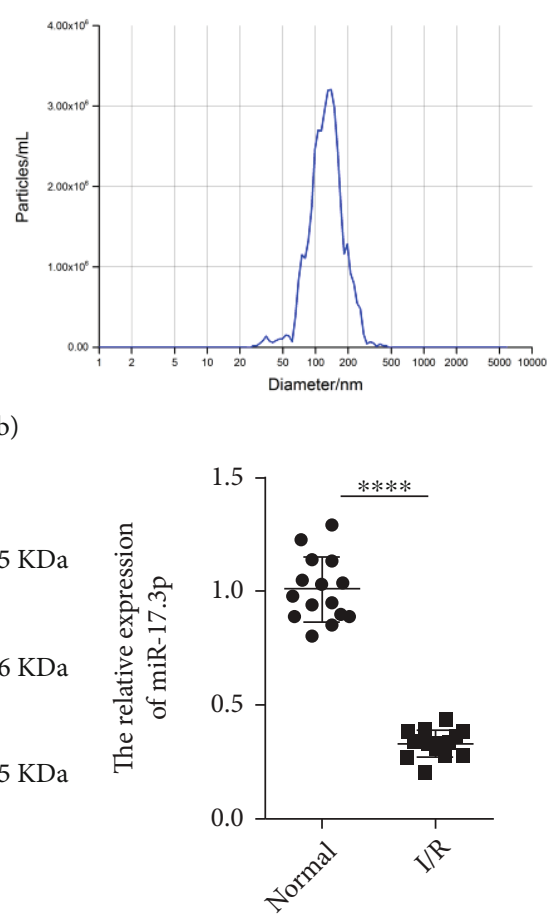

(d)

FIGURE 1: miR-17-3p was significantly downregulated in peripheral blood exosomes from patients with cardiac I/R injury. (a) The morphology of the peripheral blood exosomes was detected by TEM $(n=3$, bar $=100 \mu \mathrm{m})$. (b) The concentration and diameters of exosome particles were determined using the NTA assay $(n=3)$. (c) The expression of exosomal markers (CD63, CD9, and TSG101) was evaluated by Western blotting $(n=3)$. (d) The expression of exosomal miR-17-3p in the peripheral blood was measured by qRT-PCR $(n=15)$. U6 was used as a control. Data are presented as the mean $\pm \mathrm{SD} ;{ }^{* * * *} P<0.001, \mathrm{I} / \mathrm{R}$ injury vs. control.

spectrophotometer (BioTek Instruments Inc.). All animal experiments were performed in accordance with the Guide for the Care and Use of Laboratory Animal by International Committees. The protocol was approved by the Institutional Animal Care and Use Committee of Southeast University.

\subsection{Hematoxylin-Eosin and Masson Staining. Cardiac tissues} were fixed with $4 \%$ paraformaldehyde for $24 \mathrm{~h}$. Paraffinembedded tissues were cut into $6 \mu \mathrm{m}$ sections and dried at $45^{\circ} \mathrm{C}$. For H\&E staining, the tissues were stained with hematoxylin for $3 \mathrm{~min}$ and then placed in $1 \%$ hydrochloric acid in ethanol for $2 \mathrm{sec}$. The sections were then stained with eosin for $3 \mathrm{~min}$, dehydrated with gradient alcohol, permeabilized with xylene, sealed with resinene, and dried for $72 \mathrm{~h}$. For
Masson staining, the tissue sections were dewaxed with xylene (three times for $5 \mathrm{~min}$ each) and soaked in an alcohol gradient. The sections were stained using the Masson kit (Solarbio G1345). Changes in inflammatory infiltration and myocardial fibrosis were observed using light microscopy (Olympus Mountain, Tokyo, Japan), and images were analyzed using Image-Pro Plus software.

2.9. Statistical Analysis. Data were analyzed using SPSS 20.0 (IBM) and presented as the mean \pm SD with at least three repeats. Differences between two groups and multiple groups were determined using the Student's $t$-test and oneway ANOVA with Tukey's test, respectively. $P<0.05$ was considered statistically significant. 

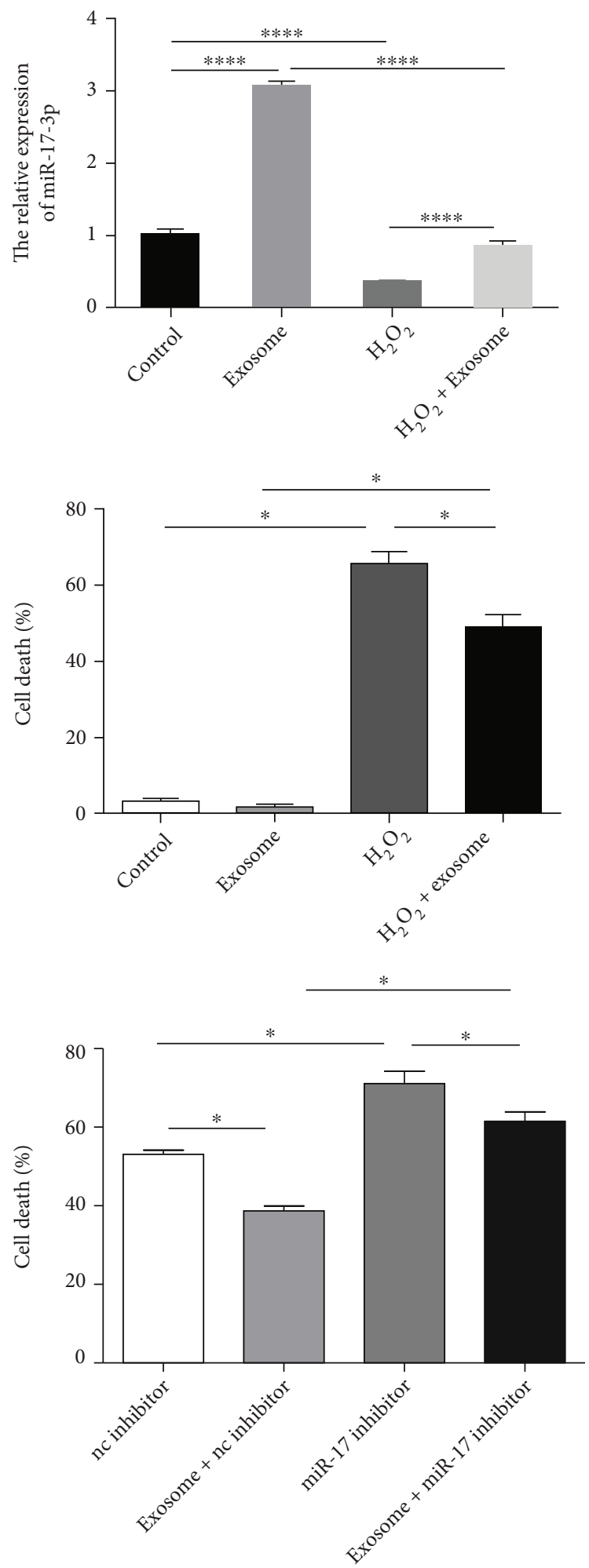

(a)

Figure 2: Continued. 

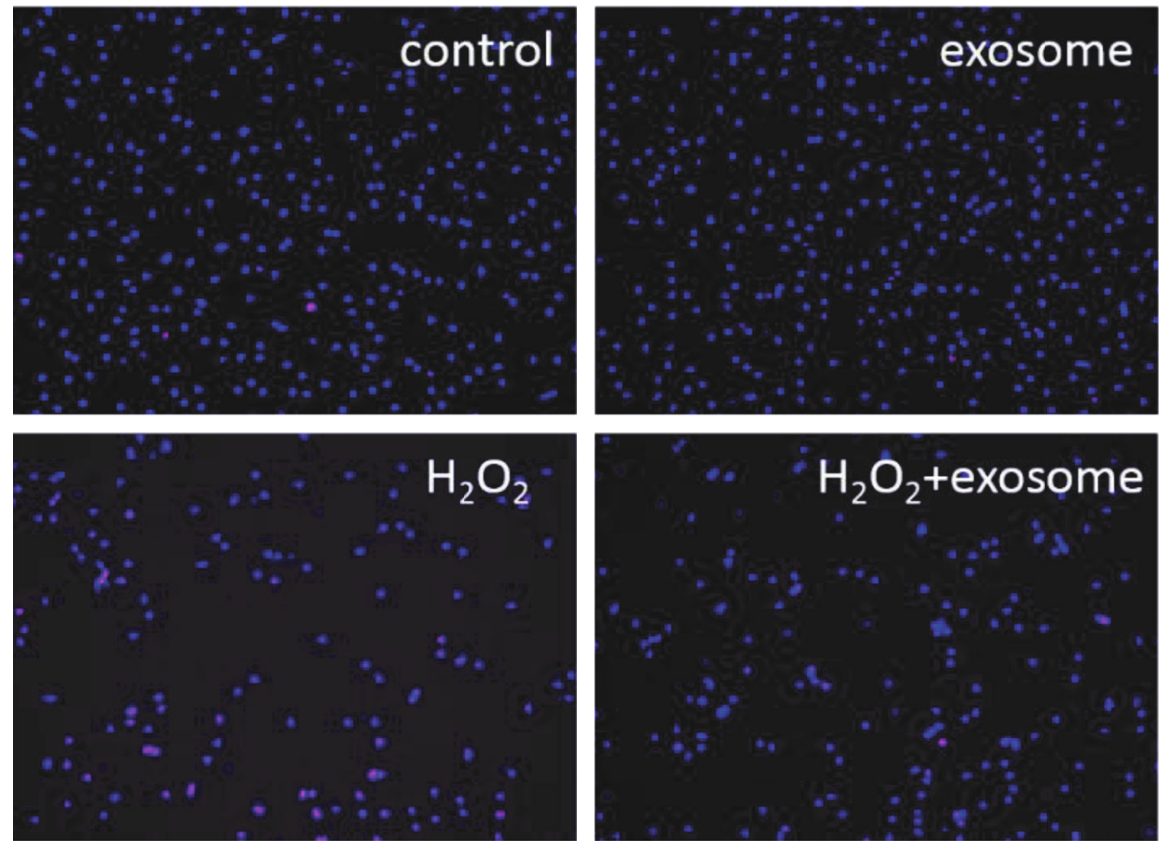

(b)
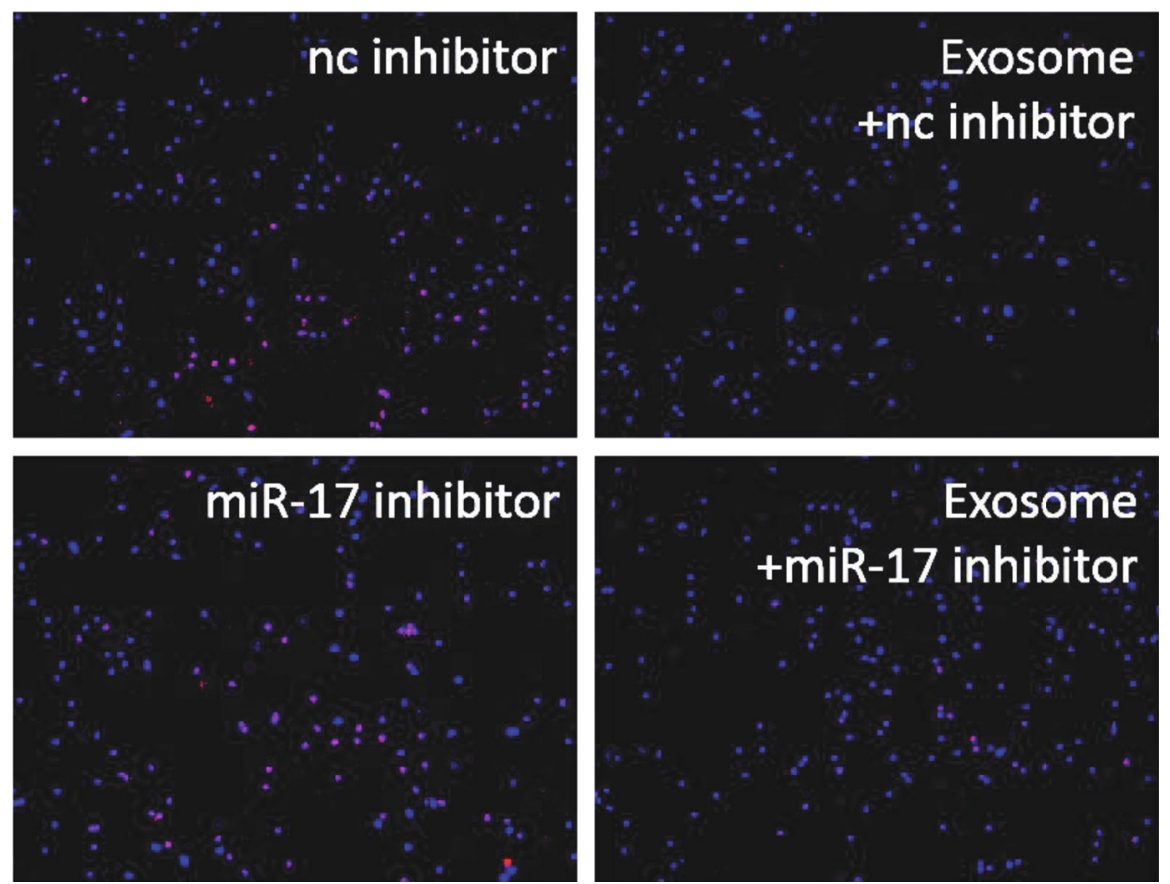

(c)

Figure 2: Exosomal miR-17-3p attenuated $\mathrm{H}_{2} \mathrm{O}_{2}$-induced programmed necrosis in primary cardiomyocytes in vitro. (a) miR-17-3p expression was measured by qRT-PCR in primary cardiomyocytes treated with peripheral blood exosomes ( $n=3)$ using U6 as the control gene. $(b, c)$ Necrotic cell death was assessed using PI staining following treatment with peripheral blood exosomes or the miR$17-3 p$ inhibitor $(n=3)$. The representative images show the PI-positive cells (bar $=100 \mu \mathrm{m})$. Data are presented as the mean \pm SD; ${ }^{*} P<$ $0.05 ;{ }^{* * *} P<0.001$

\section{Results}

3.1. Exosomal miR-17-3p Was Significantly Downregulated in the Peripheral Blood of Patients with Cardiac I/R Injury. To understand the function of exosomal miR-17-3p, exosomes were isolated from the peripheral blood of patients with cardiac I/R injury or healthy individuals. TEM analysis showed that the isolated exosomes had typical exosomal morphology, including a cup-shaped canonical appearance with a double-layer membrane (Figure 1(a)). Nanoparticle tracking analysis (NTA) showed that most particles were $100-200 \mathrm{~nm}$ in diameter, with a peak at $150 \mathrm{~nm}$ 


\begin{tabular}{|c|c|c|c|c|c|c|c|}
\hline & $\begin{array}{l}\text { Predicted consequential pairing of target region (top) } \\
\text { and miRNA (bottom) }\end{array}$ & $\begin{array}{l}\text { Site } \\
\text { type }\end{array}$ & $\begin{array}{c}\text { Context }++ \\
\text { score }\end{array}$ & $\begin{array}{l}\text { Context }++ \text { score } \\
\text { percentile }\end{array}$ & $\begin{array}{c}\text { Weighted } \\
\text { context ++ score }\end{array}$ & $\begin{array}{l}\text { Conserved branch } \\
\text { length }\end{array}$ & ${ }^{P_{C T}}$ \\
\hline $\begin{array}{l}\text { Position 1351-1357 of TIMP3 3' UTR } \\
\text { hsa-miR-17-3p }\end{array}$ & $\begin{array}{ccc}5^{\prime} & \ldots \text { GCACAGGAGGGAGAGCUGCAGAG. .. } \\
3^{\prime} & \text { GaUGUUCACGGAAGUGACGUCA }\end{array}||||||$ & $\begin{array}{c}7 \mathrm{mer} \\
\mathrm{A} 1\end{array}$ & -0.03 & 66 & -0.01 & 0.009 & N/A \\
\hline
\end{tabular}

(a)

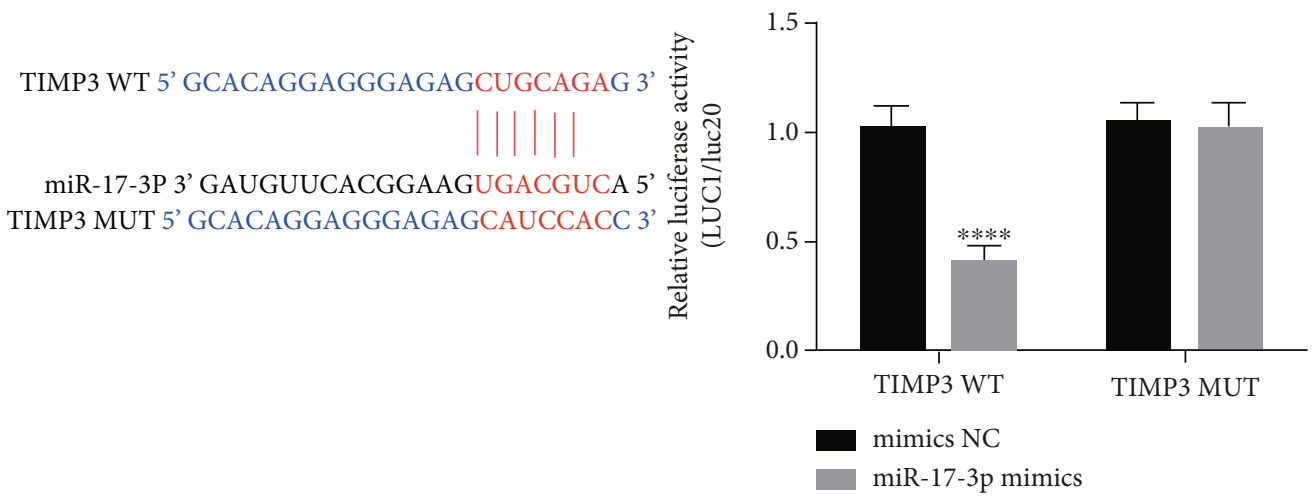

(b)

\begin{tabular}{|c|c|c|c|c|c|c|c|c|c|c|c|c|c|c|c|c|}
\hline \multirow[b]{2}{*}{ Target gene } & \multirow{2}{*}{\begin{tabular}{|c} 
Represent- \\
ative \\
transcript \\
ENST0000...
\end{tabular}} & \multirow[b]{2}{*}{ Gene name } & \multirow{2}{*}{$\begin{array}{l}\text { Number of } \\
\text { 3P-seqtags } \\
\text { supporting } \\
\text { UTR + }+5\end{array}$} & \multirow[b]{2}{*}{$\begin{array}{l}\text { Link to } \\
\text { sites in } \\
\text { UTRs }\end{array}$} & \multicolumn{4}{|c|}{ Site counts } & \multirow[b]{2}{*}{$\begin{array}{l}6 \text { mer } \\
\text { sites }\end{array}$} & \multirow[b]{2}{*}{$\begin{array}{c}\text { Representative } \\
\text { miRNA }\end{array}$} & \multicolumn{3}{|c|}{ Predicted occupancy } & \multirow{2}{*}{$\begin{array}{c}\text { Cumulative } \\
\text { weighted } \\
\text { context ++ } \\
\text { score }\end{array}$} & \multirow{2}{*}{$\begin{array}{c}\text { Total } \\
\text { context ++ } \\
\text { score }\end{array}$} & \multirow{2}{*}{$\begin{array}{l}\text { Aggregate } \\
{ }_{\mathrm{P}} \mathrm{CT}\end{array}$} \\
\hline & & & & & total & & $\mathrm{m} 8$ & A1 & & & low & $\begin{array}{l}\text { high } \\
\text { milRNA }\end{array}$ & $\begin{array}{l}\text { irins: } \\
\text { receced }\end{array}$ & & & \\
\hline $\mathrm{ZNF} 28$ & 0438150.2 & zinc finger protein 28 & 9 & Sites in UTR & 2 & 2 & 0 & 0 & 0 & hsa-miR-17-3p & N/A & N/A & $\mathrm{N} / \mathrm{A}$ & -1.16 & -1.16 & N/A \\
\hline FAM83F & 0333407.6 & family with sequence similarly 83 , member F & 5 & Sites in UTR & & 1 & 2 & 4 & 9 & hsa-miR-17-3p & $\mathrm{N} / \mathrm{A}$ & $\mathrm{N} / \mathrm{A}$ & $\mathrm{N} / \mathrm{A}$ & -0.97 & -0.97 & N/A \\
\hline NEUROG1 & 0314744.4 & neurogenin 1 & 5 & Sites in UTR & 2 & 2 & 0 & 0 & 0 & hsa-miR-17-3p & $\mathrm{N} / \mathrm{A}$ & $\mathrm{N} / \mathrm{A}$ & $\mathrm{N} / \mathrm{A}$ & -0.85 & -0.85 & N/A \\
\hline RAP2A & 0245304.4 & RAP2A, member of RAS oncogene family & 721 & Sites in UTR & & 2 & 2 & 0 & 0 & hsa-miR-17-3p & $\mathrm{N} / \mathrm{A}$ & $\mathrm{N} / \mathrm{A}$ & $\mathrm{N} / \mathrm{A}$ & -0.85 & -0.89 & $\mathrm{~N} / \mathrm{A}$ \\
\hline КСтD9 & 0221200.4 & potassium channel tetramerization domain containing 9 & 575 & Sites in UTR & 2 & 2 & 0 & 0 & 1 & hsa-miR-17-3p & $\mathrm{N} / \mathrm{A}$ & $\mathrm{N} / \mathrm{A}$ & $\mathrm{N} / \mathrm{A}$ & -0.81 & -0.81 & N/A \\
\hline MTCPI & 0369476.3 & mature T-cell proliferation 1 & 18 & Sites in UTR & 2 & 1 & 1 & 0 & 1 & hsa-miR-17-3p & $\mathrm{N} / \mathrm{A}$ & $\mathrm{N} / \mathrm{A}$ & $\mathrm{N} / \mathrm{A}$ & -0.78 & -0.78 & N/A \\
\hline SCGB1D2 & 0244926.3 & secretogoblin, family ID, member 2 & 5 & Sites in UTR & 1 & 1 & 0 & 0 & 0 & hsa-miR-17-3p & N/A & $\mathrm{N} / \mathrm{A}$ & $\mathrm{N} / \mathrm{A}$ & 0.73 & 0.73 & N/A \\
\hline SCGBID1 & 0306238.3 & secretogoblin family $1 \mathrm{D}$, member 1 & 5 & Sites in UTR & 1 & 1 & 0 & 0 & 0 & hsa-miR-17-3p & $\mathrm{N} / \mathrm{A}$ & $\mathrm{N} / \mathrm{A}$ & $\mathrm{N} / \mathrm{A}$ & -0.73 & -0.73 & $\mathrm{~N} / \mathrm{A}$ \\
\hline KCNMA1 & 0481070.1 & $\begin{array}{l}\text { potassium large conductance calcium-activated channel, } \\
\text { subfamily M, alpha member } 1\end{array}$ & 12 & Sites in UTR & 1 & 1 & 0 & 0 & 0 & hsa-miR-17-3p & $\mathrm{N} / \mathrm{A}$ & $\mathrm{N} / \mathrm{A}$ & $\mathrm{N} / \mathrm{A}$ & -0.71 & -0.71 & $\mathrm{~N} / \mathrm{A}$ \\
\hline GPD2 & 0540309.1 & glycerol-3-phosphate dehydrogenase 2 (mitrochondrial) & 195 & Sites in UTR & 3 & 3 & 0 & 0 & 0 & hsa-miR-17-3p & $\mathrm{N} / \mathrm{A}$ & $\mathrm{N} / \mathrm{A}$ & $\mathrm{N} / \mathrm{A}$ & -0.69 & -0.70 & N/A \\
\hline
\end{tabular}

(c)
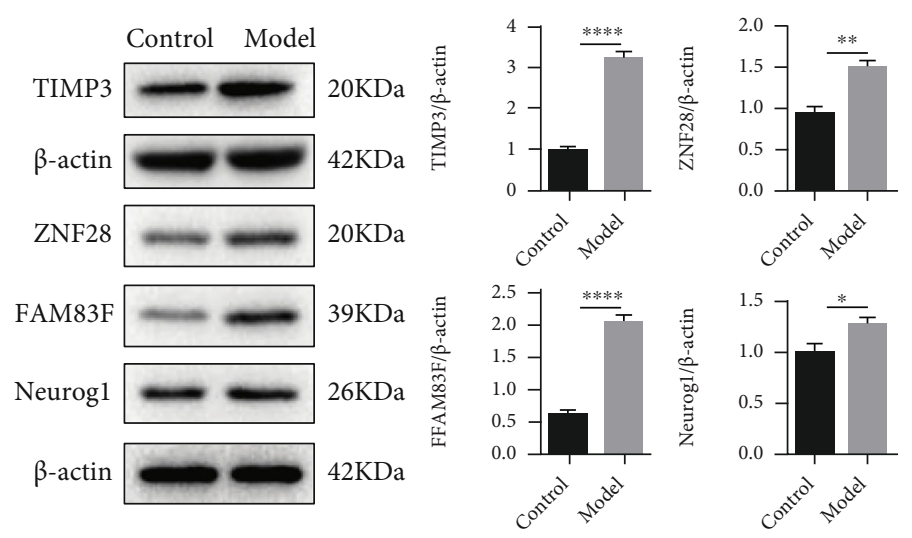

(d)

Figure 3: Continued. 

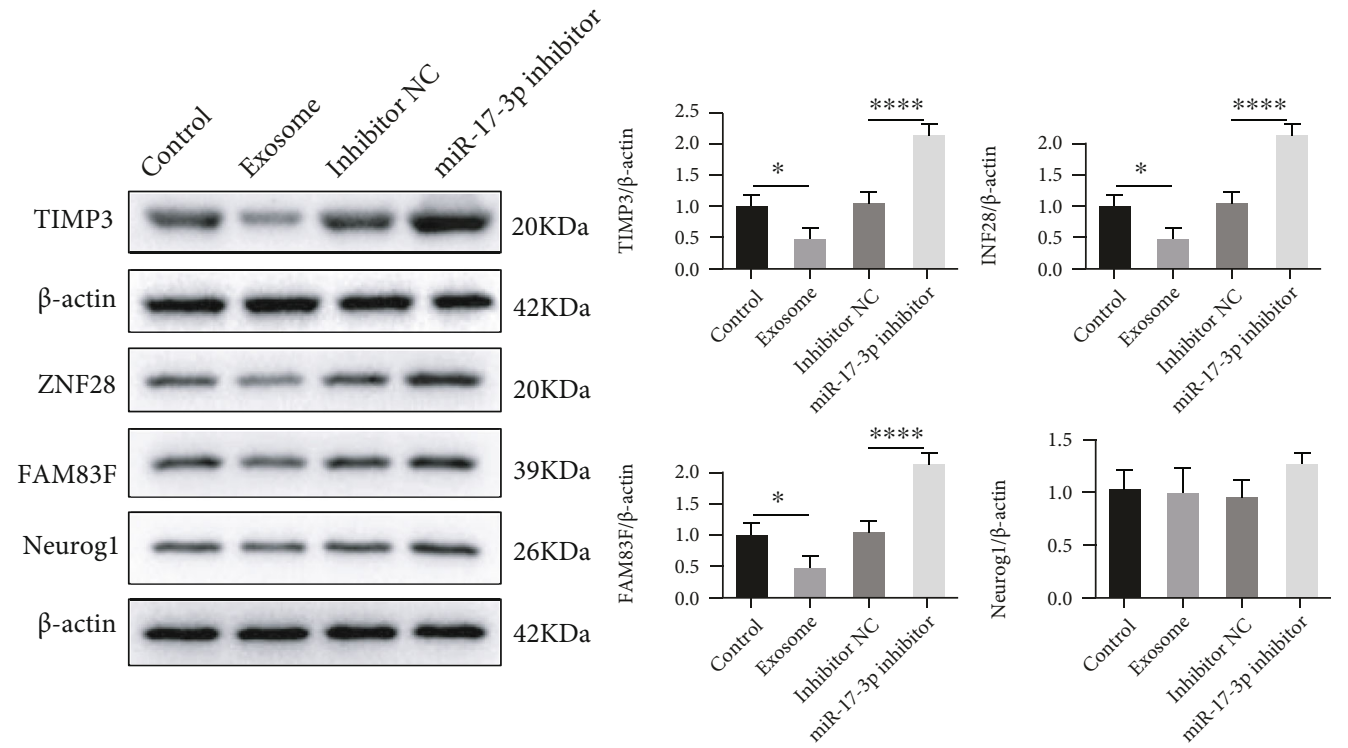

(e)

FIgURE 3: TIMP3 is a target of miR-17-3p. (a) The miR-17-3p binding site in the TIMP3 $3{ }^{\prime}$ UTR was predicted using TargetScan7.2. (b) Dual-luciferase reporter assay was used to detect the luciferase activity cotransfected with $3^{\prime}$ UTR of TIMP3 with the wild-type binding site or mutant binding site and miR-17-3p mimic. (c) The miR-17-3p targets were predicted with TargetScan7.2. (d) ZNF28, FAM83F, Neurog1, and TIMP3 levels in the cardiac I/R injury model were determined by Western blotting. Data are presented as the mean \pm SD; ${ }^{* *} P<0.01$, model vs. control. (e) The effect of miR-17-3p on ZNF28, FAM83F, Neurog1, and TIMP3 levels was determined by Western blot analysis. The data are presented as the mean $\pm \mathrm{SD}(n=3) ;{ }^{*} P<0.05 ;{ }^{* * * *} P<0.001$.

(Figure 1(b)). In addition, Western blotting revealed that the exosomal markers CD63, CD9, and TSG101 were highly expressed (Figure 1(c)), indicating that exosomes were successfully isolated from the peripheral blood. To understand the function of miR-17-3p, miR-17-3p expression levels in the exosomes from the different samples were measured by qRT-PCR. As shown in Figure 1(d), miR-17-3p expression was significantly decreased in the peripheral blood exosomes from patients with cardiac I/R injury compared to healthy individuals. These results demonstrated that exosomal miR$17-3 p$ in peripheral blood might play a crucial role in cardiac I/R injury.

3.2. Exosomal miR-17-3p Attenuated $\mathrm{H}_{2} \mathrm{O}_{2}$-Induced Programmed Necrosis in Primary Cardiomyocytes In Vitro. The effect of exosomal miR-17-3p on $\mathrm{H}_{2} \mathrm{O}_{2}$-induced programmed necrosis in primary cardiomyocytes was investigated by treating the cells with exosomes. As shown in Figure 2(a), miR-17-3p expression was significantly elevated following the treatment of control or $\mathrm{H}_{2} \mathrm{O}_{2}$-induced primary cardiomyocytes with peripheral blood exosomes. miR-17-3p expression was much lower in $\mathrm{H}_{2} \mathrm{O}_{2}$-induced primary cardiomyocytes than in the control cells. Moreover, the number of primary cardiomyocytes undergoing programmed necrosis was dramatically increased following treatment with $\mathrm{H}_{2} \mathrm{O}_{2}$; however, treatment with exosomes significantly reduced the number of $\mathrm{H}_{2} \mathrm{O}_{2}$-induced necrotic primary cardiomyocytes (Figure 2(b)). Furthermore, inhibition of miR17-3p expression in $\mathrm{H}_{2} \mathrm{O}_{2}$-induced primary cardiomyocytes by transfection with a miR-17-3p inhibitor significantly increased the number of necrotic primary cardiomyocytes (inhibitor vs. NC), which was partially rescued by treatment with exosomes (Figure 2(c)). These results suggested that exosomal miR-17-3p attenuated $\mathrm{H}_{2} \mathrm{O}_{2}$-induced programmed necrosis of primary cardiomyocytes in vitro.

3.3. TIMP3 Is a Direct Target for miR-17-3p. We have demonstrated that miR-17-3p could regulate TIMP3 expression. Using TargetScan, we predicted the binding site for miR-17$3 p$ in the $3^{\prime}$ UTR of TIMP3 (Figure 3(a)). Dual-luciferase reporter gene assay showed that the luciferase activity was decreased in myocardium cells cotransfected with $3^{\prime}$ UTR of TIMP3 plasmid containing a wild-type binding site and miR-17-3p mimic compared with the miR-17-3p mimic NC group (Figure 3(b)). To further confirm the importance of TIMP3, we predicted the targets of miR-17-3p using TargetScan. The results showed that ZNF28, FAM83F, and Neurog1 had the highest total context++ score (Figure 3(c)). Moreover, ZNF28, FAM83F, Neurog1, and TIMP3 expression levels were significantly elevated in the myocardial I/R injury mice compared to the control mice (Figure $3(\mathrm{~d})$ ). In addition, the exosomal miR-17-3p mimic and inhibitor significantly decreased and elevated ZNF28, FAM83F, and TIMP3 expression, in the cardiomyocytes, with TIMP3 having the highest fold changes in response to miR-17-3p modulation (Figure 3(e)). Taken together, exosomal miR-17-3p could negatively regulate the expression of its target genes, especially TIMP3.

3.4. TIMP3 Affected $\mathrm{H}_{2} \mathrm{O}_{2}$-Induced Programmed Necrosis of Cardiomyocytes Regulated by miR-17-3p In Vitro. To understand the role of TIMP3 on programmed necrosis, the effect of TIMP3 overexpression and knockdown on $\mathrm{H}_{2} \mathrm{O}_{2}$-induced 


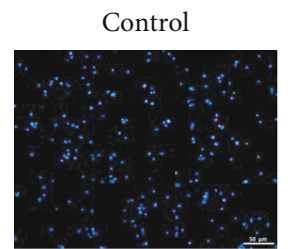

$\mathrm{H}_{2} \mathrm{O}_{2}+$ Over-TIMP3

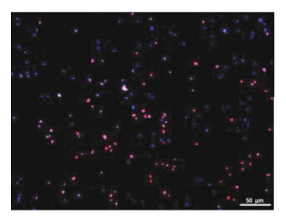

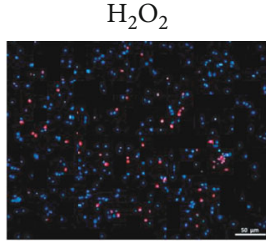

$\mathrm{H}_{2} \mathrm{O}_{2}+\mathrm{Si}-\mathrm{NC}$

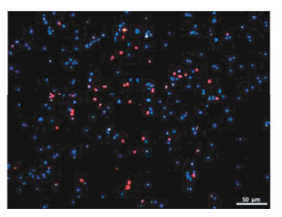

$\mathrm{H}_{2} \mathrm{O}_{2}+$ Over-NC

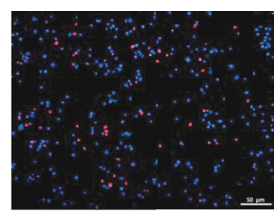

$\mathrm{H}_{2} \mathrm{O}_{2}+\mathrm{Si}$-TIMP3

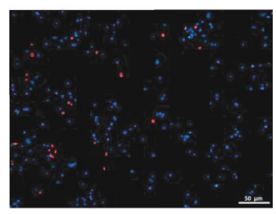

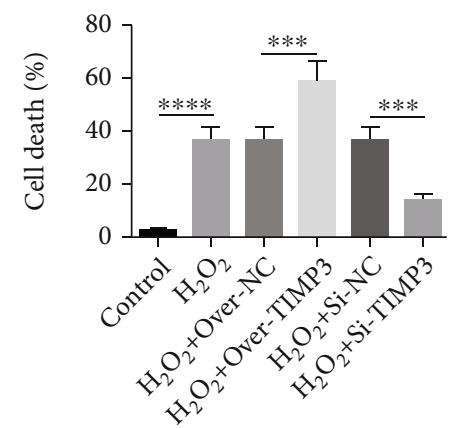

(a)

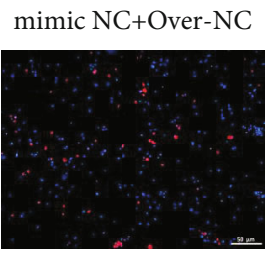

mimic NC+OverTIMP3

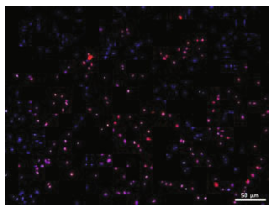

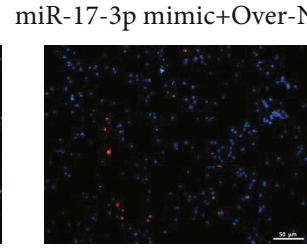

miR-17-3p mimic +Over-TIMP3

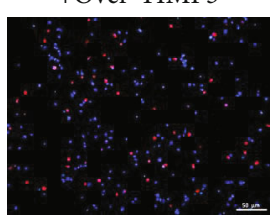

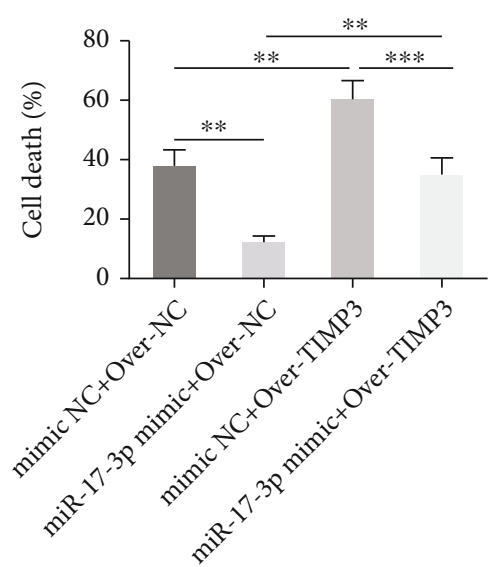

(b)

FIGURE 4: TIMP3 affected $\mathrm{H}_{2} \mathrm{O}_{2}$-induced programmed necrosis of primary cardiomyocytes and was regulated by miR-17-3p in vitro. (a) Necrotic cell death was assessed using PI staining of H9C2 cells following TIMP3 overexpression or knockdown. (b) The function of TIMP3 in necrotic cell death was identified using a rescue experiment. Representative images show PI-positive cells $($ bar $=100 \mu \mathrm{m})$. Data are presented as the mean $\pm \mathrm{SD}(n=3) ;{ }^{* *} P<0.01 ;{ }^{* * *} P<0.001 ;{ }^{* * * *} P<0.0001$.

primary cardiomyocytes was investigated. We found that the number of primary cardiomyocytes undergoing programmed necrosis was dramatically increased following treatment with $\mathrm{H}_{2} \mathrm{O}_{2}$. This effect was aggravated by TIMP3 overexpression (Figure 4(a)). In contrast, programmed necrosis was greatly reduced by TIMP3 knockdown, suggesting that TIMP3 upregulation promoted $\mathrm{H}_{2} \mathrm{O}_{2}$-induced programmed necrosis in primary cardiomyocytes in vitro. To determine whether the function of TIMP3 in $\mathrm{H}_{2} \mathrm{O}_{2}$-induced programmed necrosis was affected by $\mathrm{miR}-17-3 \mathrm{p}$, a rescue experiment was performed. The results demonstrated that the elevated number of necrotic cells observed in TIMP3-overexpressing cells could be partially reduced by treatment with miR-17-3p-expressing exosomes (Figure 4(b)). These results demonstrated that TIMP3 could increase $\mathrm{H}_{2} \mathrm{O}_{2}$-induced programmed necrosis of primary cardiomyocytes and this function could be regulated by $\mathrm{miR}-17-3 \mathrm{p}$ in vitro.

3.5. Exosomal miR-17-3p Greatly Alleviated Cardiac I/R Injury. To further investigate the effects of exosomal miR17-3p on cardiac I/R injury, a cardiac $I / R$ injury mouse model was established. These mice were treated with exosomes or the miR-17-3p inhibitor. miR-17-3p expression was significantly increased following exosome treatment but decreased after treatment with the miR-17-3p inhibitor (Figure 5(a)). Echocardiography analysis showed that LVEDd and LVEDs were significantly elevated while EF (\%) of the left ventricle and fractional shortening (FS) (\%) at the left ventricular short axis were significantly decreased in the cardiac I/R injury model compared to the control group. However, treatment with exosomes resolved these changes. In contrast, treatment with the miR-17-3p inhibitor further aggravated these parameters in the cardiac I/R injury mice (Figure 5(b)). TTC staining showed that the infarct size was significantly increased in the cardiac I/R injury mice compared to the control mice. Consistent with these echocardiography results, the infarct size decreased following exosome treatment and further increased in the presence of the exosomal miR-17-3p inhibitor (Figure 5(c)). H\&E and Masson staining indicated that inflammatory cell infiltration and fibrosis were increased in the injury model. However, these increases were dramatically reduced 


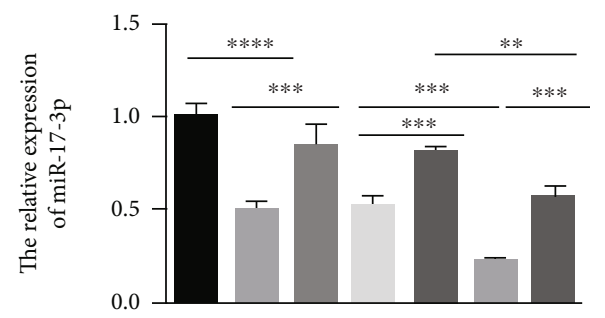

(a)
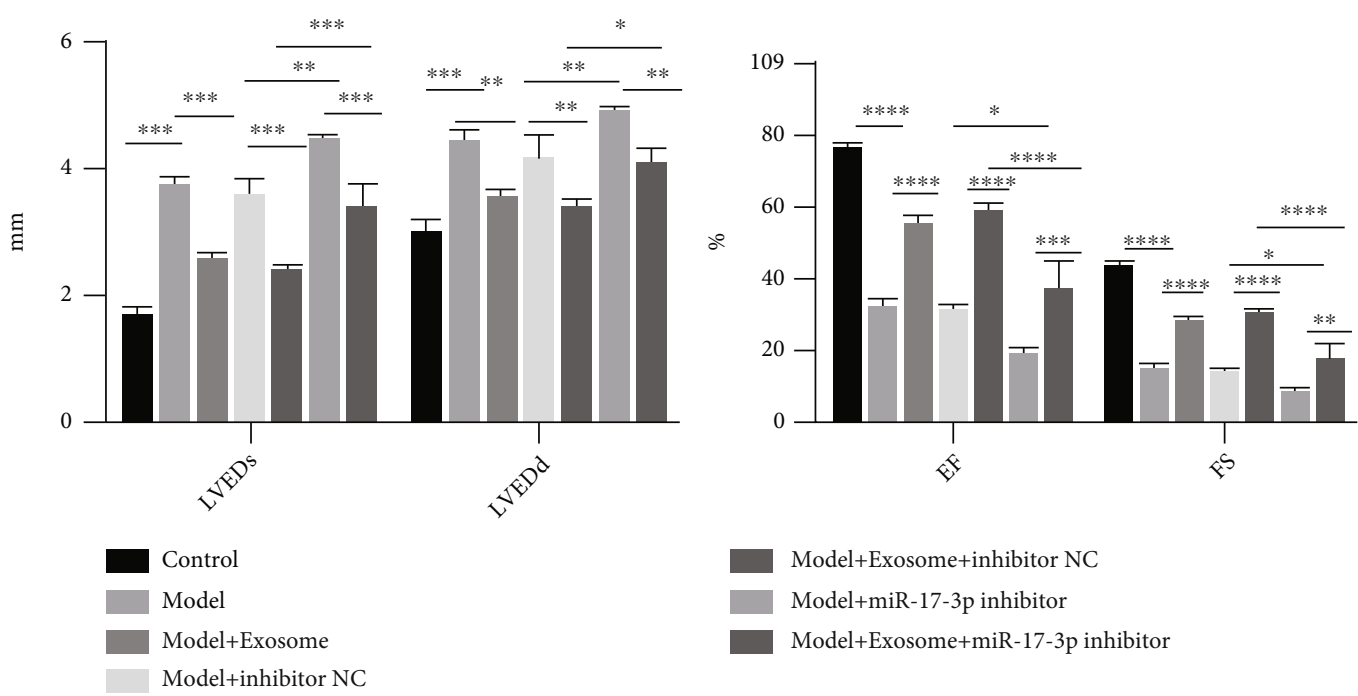

(b)

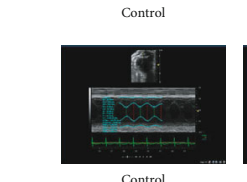

Model
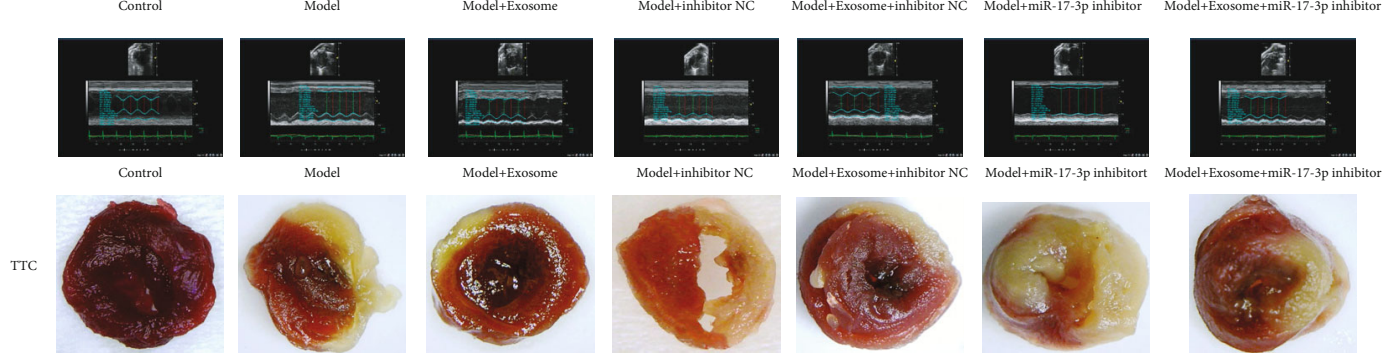

(c)
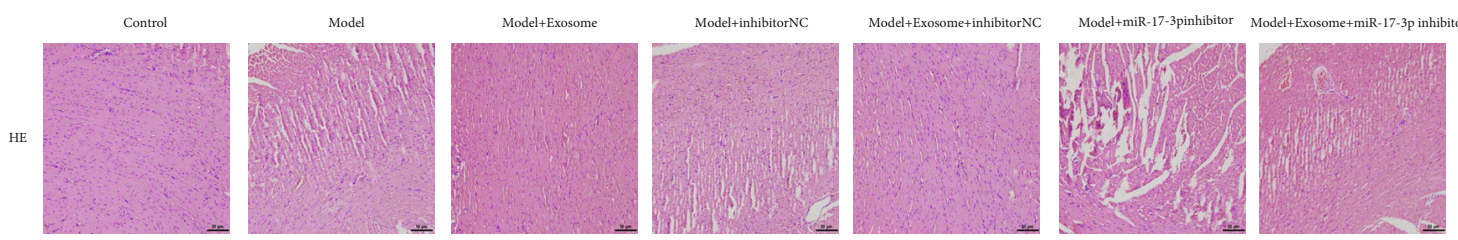

(d)
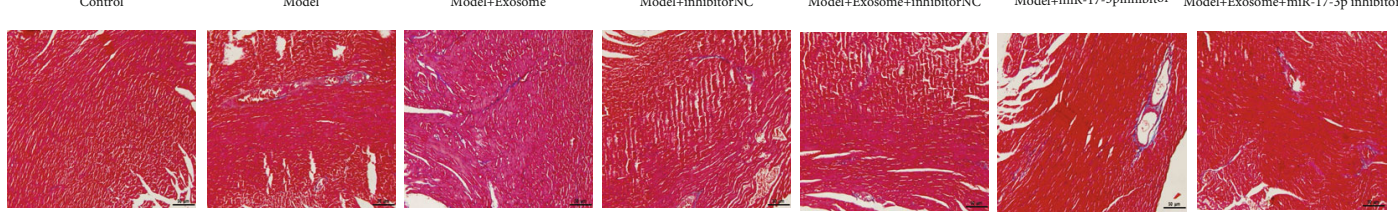

(e)

Figure 5: Exosomal miR-17-3p greatly alleviated cardiac I/R injury. (a) miR-17-3p expression was measured by qRT-PCR. (b) LVEDd, LVEDs, and EF of the left ventricle and fractional shortening (FS) at the left ventricular short axis after cardiac I/R injury were evaluated by echocardiography. (c) The effects of the exosomes and miR-17-3p inhibitor on the infarct size were determined by TTC staining.(d, e) The effect of exosomal miR-17-3p on inflammatory cell infiltration and fibrosis was investigated using H\&E and Masson staining, respectively $($ bar $=100 \mu \mathrm{m})$. Data are presented as the mean $\pm \mathrm{SD}(n=3) ;{ }^{* *} P<0.01 ;{ }^{* * *} P<0.001 ;{ }^{* * * *} P<0.0001$. 


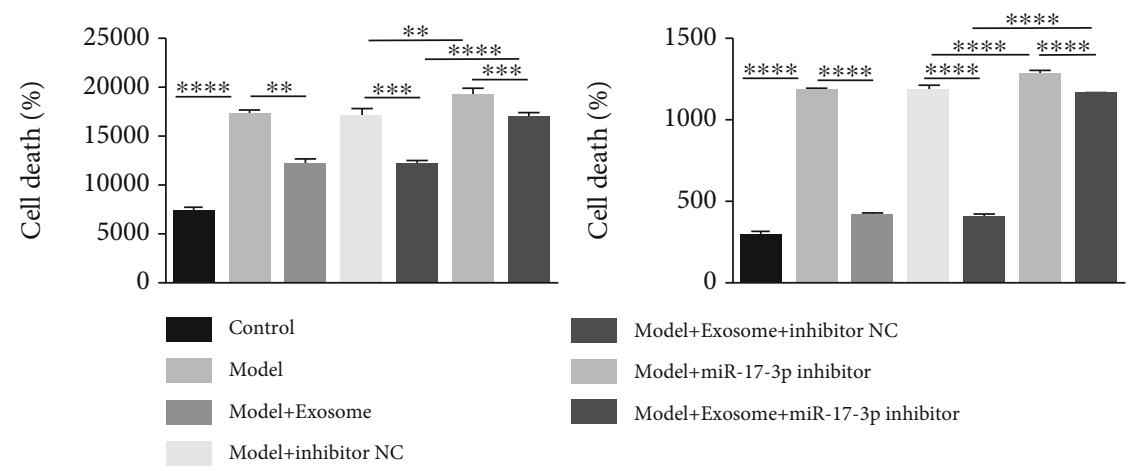

(a)
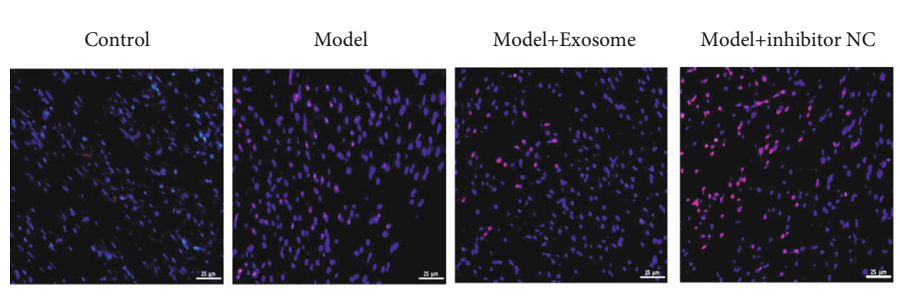

Model+Exosome+ inhibitor NC

Model+miR-17-3
inhibitor

Model+Exosome+miR
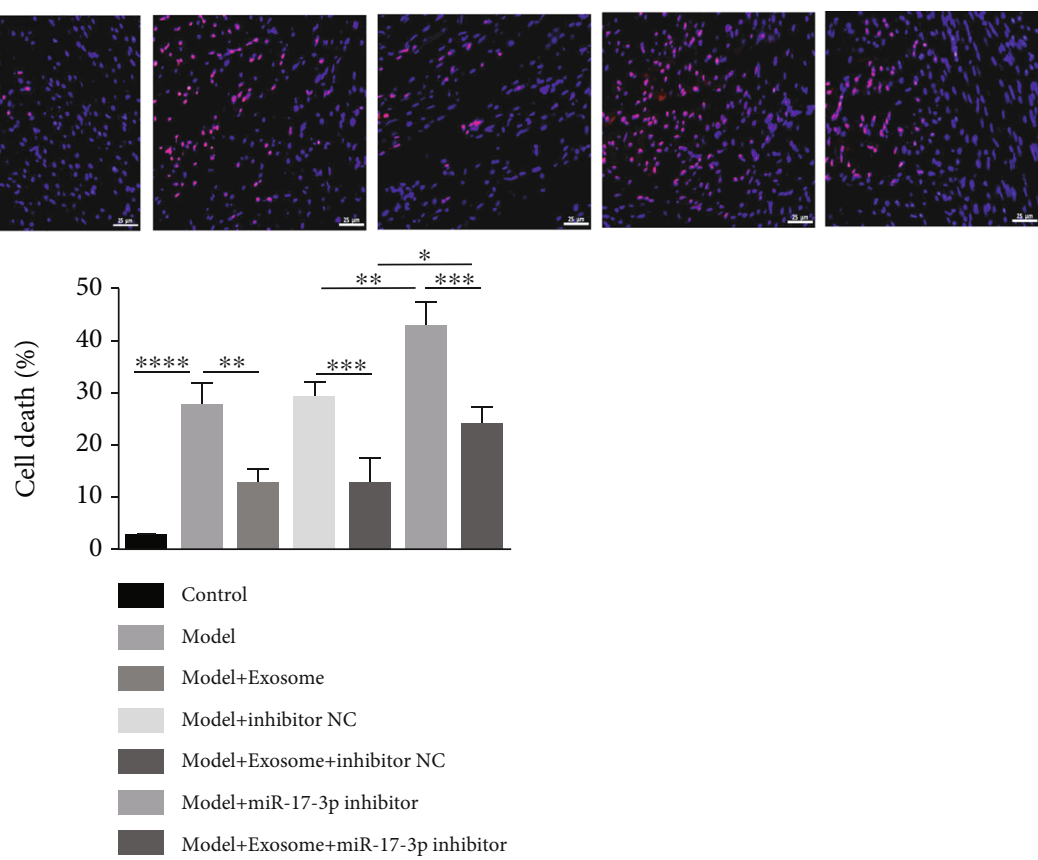

(b)

Figure 6: Exosomal miR-17-3p affected the LDH and TNF- $\alpha$ levels and necrotic cell death. (a) LDH and TNF- $\alpha$ levels were affected by exosomal miR-17-3p. (b) The effect of exosomal miR-17-3 on necrotic cell death was determined by PI staining (bar $=100 \mu \mathrm{m}$ ). Data are presented as the mean \pm SD $(n=3) ;{ }^{* *} P<0.01 ;{ }^{* * *} P<0.001 ;{ }^{* * *} P<0.0001$.

following exosome treatment. In contrast, treatment with the exosomal miR-17-3p inhibitor exacerbated inflammatory cell infiltration (Figures 5(d) and 5(e)).

To confirm these results, ventricular remodeling-related gene $(\alpha-\mathrm{MHC}, \beta$-MHC, ANP, and BNP) and fibrosisrelated gene ( $\alpha$-SMA, Col $3 \alpha 1$, and Col $1 \alpha 1)$ expression levels were measured by qRT-PCR. The results showed that $\alpha$ SMA, Col $1 \alpha 1$, Col $3 \alpha 1$, ANP, BNP, and $\beta$-MHC expression levels were significantly elevated, while $\alpha$-MHC expression levels were significantly decreased in the cardiac I/R injury model group compared with the control group. However, treatment with exosomes could partially rescue these expression changes. In contrast, the expression levels of $\alpha$-SMA, $\operatorname{Col} 1 \alpha 1, \operatorname{Col} 3 \alpha 1, \mathrm{ANP}, \mathrm{BNP}$, and $\beta$-MHC were further increased, while $\alpha$-MHC levels decreased after treatment with the exosomal miR-17-3P inhibitor (Supplementary Figure 1). Moreover, $\mathrm{LDH}$ and $\mathrm{TNF}-\alpha$ levels were dramatically increased in the model group and further elevated following treatment with the exosomal miR-17-3p inhibitor. In contrast, the LDH and TNF- $\alpha$ levels significantly decreased following treatment with exosomes (Figure 6(a)). In addition, necrotic cells were significantly elevated in the model group compared to the control mice. Furthermore, the number of necrotic cells in model mice dramatically decreased following exosome treatment but they were further increased after treatment with the miR-17-3p inhibitor (Figure 6(b)). These results demonstrated that exosomal miR-17-3p greatly alleviated cardiac I/R injury. 


\section{Discussion}

Myocardial I/R injury is a common pathophysiological process in ischemic heart disease and an important cause of aggravated myocardial injury. Programmed necrosis plays a crucial role in myocardial I/R injury. However, how exosomal miRNAs are regulated in programmed necrosis during myocardial $\mathrm{I} / \mathrm{R}$ injury remains unclear. The present study demonstrated that exosomal miR-17-3p from peripheral blood alleviated cardiac $\mathrm{I} / \mathrm{R}$ injury by inhibiting programmed necrosis via the miR-17-3p/TIMP3 axis.

Exosomes are nanoscale extracellular vesicles secreted by living cells and widely present in biological fluids. Exosomes carry proteins, lipids, and nucleic acids that can reflect the physiological and pathological conditions of the source cells. They play an important role in the exchange of material and information between cells [21]. An increasing number of studies have demonstrated that exosomal miRNAs could participate in myocardial I/R injury [21]. Exosomes or microvesicles from induced pluripotent stem cells deliver cardioprotective miRNAs and prevent cardiomyocyte apoptosis in the ischemic myocardium [22]. Plasma exosomes induced by remote ischemic preconditioning attenuate myocardial I/R injury by transferring with miR-24 [23]. In a previous study, miR-17-3p greatly contributed to exercise-induced cardiac growth and protected against myocardial I/R injury. However, the function of exosomal miR-17-3p in myocardial $\mathrm{I} / \mathrm{R}$ injury remains unclear. In the present study, we found that miR-17-3p was significantly downregulated in peripheral blood exosomes from patients with cardiac I/R injury, suggesting that exosomal miR-17-3p might play an important role in myocardial I/R injury.

Multiple studies have demonstrated that programmed necrosis is involved in myocardial I/R injury [12]. Xu et al. [19] demonstrated that inhibition of MPTP opening regulates programmed necrosis and myocardial I/R injury. Sun et al. [24] showed that parkin regulates programmed necrosis and myocardial I/R injury by targeting cyclophilin-D. In addition, Wang et al. [20] found that the long noncoding RNA NRF could regulate programmed necrosis and myocardial injury during ischemia and reperfusion by targeting miR- 873 . MicroRNA-103 and microRNA-107 regulate programmed necrosis and myocardial I/R injury through targeting FADD [14]. These findings suggest that exosomal miRNAs can modulate myocardial I/R injury by regulating programmed necrosis. In the present study, we found that exosomal miR-17-3p attenuated $\mathrm{H}_{2} \mathrm{O}_{2}$-induced programmed necrosis of cardiomyocytes in vitro and exosomal miR-17-3p greatly alleviated cardiac I/R injury by inhibiting programmed necrosis in vivo.

miRNAs are involved in mRNA translation or degradation by binding the $3^{\prime}$ UTR of mRNA, thereby achieving posttranscriptional gene regulation. Many miRNA targets are involved in cardiac I/R injury, including microRNA193b/mastermind-like 1, miR-346/Bax, and miR-24-3p/ RIPK1 $[7,25,26]$. In the present study, we found that TIMP3 was a direct target of miR-17-3p, consistent with a previous report [15]. Several studies have indicated that TIMP3 plays an important role in myocardial I/R injury. Specifically, Wang and Tong found that precondition of sevoflurane upregulates TIMP3 expression to alleviate myocardial I/R injury [27]. Purcell et al. showed that a matrix metalloproteinase-responsive hydrogel releasing TIMP-3 after myocardial infarction affected left ventricular remodeling [28]. Here, we demonstrated that exosomal miR-17-3p alleviated programmed necrosis during cardiac I/R injury by regulating TIMP3 expression. Liu et al. showed that TIMP3 overexpression protects against cardiac I/R injury by inhibiting myocardial apoptosis through the ROS/MAPK pathway [29]. Fuji et al. found that TIMP3 deficiency disrupts the hepatocyte E-cadherin/ $\beta$-catenin complex and induces cell death in liver I/R injury [30]. These results suggested that TIMP3 might affect programmed necrosis in cardiac I/R injury by regulating ROS/MAPKs or cadherin/ $\beta$-catenin pathways. However, the signaling pathway affected by TIMP3 needs to be further studied.

\section{Conclusions}

In summary, we demonstrated that miR-17-3p is significantly downregulated in peripheral blood exosomes from patients with cardiac I/R injury. The mechanism by which exosomal miR-17-3p alleviated programmed necrosis in cardiac I/R injury was through regulating TIMP3 expression. These findings could represent a potential treatment for I/R injury.

\section{Data Availability}

The data used to support the findings of this study are included within the article.

\section{Conflicts of Interest}

The authors declare that there is no conflict of interest regarding the publication of this paper.

\section{Acknowledgments}

This work was supported by the Natural Science Foundation of Jiangsu Province (BK20190352) and the major research plan of the Natural Science Foundation of Jiangsu Province (BE2021735).

\section{Supplementary Materials}

Supplementary Figure 1: exosomal miR-17-3p affected the expression of ventricular remodeling- and fibrosis-related genes. The expression levels of ventricular remodeling and fibrosis-related genes (e.g., $\alpha$-SMA, $\operatorname{Col} 3 \alpha 1, \operatorname{Col} 1 \alpha 1, \alpha$ MHC, $\beta$-MHC, ANP, and BNP) were measured by qRTPCR using GAPDH as the control gene. Data are presented as the mean $\pm \mathrm{SD}(n=3) ;{ }^{* *} P<0.01 ;{ }^{* * *} P<0.001 ;{ }^{* * * *} P<$ 0.0001. (Supplementary Materials)

\section{References}

[1] A. Frank, M. Bonney, S. Bonney, L. Weitzel, M. Koeppen, and T. Eckle, "Myocardial ischemia reperfusion injury: from basic science to clinical bedside," Seminars in Cardiothoracic and Vascular Anesthesia, vol. 16, no. 3, pp. 123-132, 2012. 
[2] J. Ding, Z. Yang, H. Ma, and H. Zhang, "Mitochondrial aldehyde dehydrogenase in myocardial ischemic and ischemiareperfusion injury," Advances in Experimental Medicine and Biology, vol. 1193, pp. 107-120, 2019.

[3] L. Li, X. Li, Z. Zhang, L. Liu, Y. Zhou, and F. Liu, "Protective mechanism and clinical application of hydrogen in myocardial ischemia-reperfusion injury," Pakistan Journal of Biological Sciences, vol. 23, no. 2, pp. 103-112, 2020.

[4] M. Y. Wu, G. T. Yiang, W. T. Liao et al., "Current mechanistic concepts in ischemia and reperfusion injury," Cellular Physiology and Biochemistry, vol. 46, no. 4, pp. 1650-1667, 2018.

[5] Z. Q. Huang, W. Xu, J. L. Wu, X. Lu, and X. M. Chen, "MicroRNA-374a protects against myocardial ischemia-reperfusion injury in mice by targeting the MAPK6 pathway," Life Sciences, vol. 232, article 116619, 2019.

[6] S. Y. Yu, B. Dong, Z. F. Fang, X. Q. Hu, L. Tang, and S. H. Zhou, "Knockdown of lncRNA AK139328 alleviates myocardial ischaemia/reperfusion injury in diabetic mice via modulating miR-204-3p and inhibiting autophagy," Journal of Cellular and Molecular Medicine, vol. 22, no. 10, pp. 4886-4898, 2018.

[7] J. Zhang, J. Niu, B. Tian, and M. Zhao, "MicroRNA-193b protects against myocardial ischemia-reperfusion injury in mouse by targeting mastermind-like 1," Journal of Cellular Biochemistry, vol. 120, no. 8, pp. 14088-14094, 2019.

[8] Z. Wei, S. Qiao, J. Zhao et al., "miRNA-181a over-expression in mesenchymal stem cell-derived exosomes influenced inflammatory response after myocardial ischemia-reperfusion injury," Life Sciences, vol. 232, article 116632, 2019.

[9] J. Zhao, X. Li, J. Hu et al., "Mesenchymal stromal cell-derived exosomes attenuate myocardial ischaemia-reperfusion injury through miR-182-regulated macrophage polarization," Cardiovascular Research, vol. 115, no. 7, pp. 1205-1216, 2019.

[10] T. Geng, Z. Y. Song, J. X. Xing, B. X. Wang, S. P. Dai, and Z. S. $\mathrm{Xu}$, "Exosome derived from coronary serum of patients with myocardial infarction promotes angiogenesis through the miRNA-143/IGF-IR pathway," International Journal of Nanomedicine, vol. Volume 15, pp. 2647-2658, 2020.

[11] E. H. Kim, S. W. Wong, and J. Martinez, "Programmed necrosis and disease:we interrupt your regular programming to bring you necroinflammation," Cell Death and Differentiation, vol. 26, no. 1, pp. 25-40, 2019.

[12] H. Zhu and A. Sun, "Programmed necrosis in heart disease: molecular mechanisms and clinical implications," Journal of Molecular and Cellular Cardiology, vol. 116, pp. 125-134, 2018.

[13] D. Y. Zhang, B. J. Wang, M. Ma, K. Yu, Q. Zhang, and X. W. Zhang, "MicroRNA-325-3p protects the heart after myocardial infarction by inhibiting RIPK3 and programmed necrosis in mice," BMC Molecular Biology, vol. 20, no. 1, p. 17, 2019.

[14] J. X. Wang, X. J. Zhang, Q. Li et al., "MicroRNA-103/107 regulate programmed necrosis and myocardial ischemia/reperfusion injury through targeting FADD," Circulation Research, vol. 117, no. 4, pp. 352-363, 2015.

[15] J. Shi, Y. Bei, X. Kong et al., "miR-17-3p contributes to exercise-induced cardiac growth and protects against myocardial ischemia-reperfusion injury," Theranostics, vol. 7, no. 3, pp. 664-676, 2017.

[16] X. Wang, J. Chen, and X. Huang, "Rosuvastatin attenuates myocardial ischemia-reperfusion injury via upregulatingmiR-17-3pmediated autophagy," Cellular Reprogramming, vol. 21, no. 6, pp. 323-330, 2019.
[17] T. Yuan, Z. Yang, S. Xian et al., "Dexmedetomidine-mediated regulation of miR-17-3p in H9C2 cells after hypoxia/reoxygenation injury," Experimental and Therapeutic Medicine, vol. 20, no. 2, pp. 917-925, 2020.

[18] Z. Liu, Z. Zhang, J. Yao et al., "Serum extracellular vesicles promote proliferation of H9C2 cardiomyocytes by increasing miR-17-3p," Biochemical and Biophysical Research Communications, vol. 499, no. 3, pp. 441-446, 2018.

[19] T. Xu, W. Ding, X. Ao et al., "ARC regulates programmed necrosis and myocardial ischemia/reperfusion injury through the inhibition of mPTP opening," Redox Biology, vol. 20, pp. 414-426, 2019.

[20] K. Wang, F. Liu, C. Y. Liu et al., "The long noncoding RNA NRF regulates programmed necrosis and myocardial injury during ischemia and reperfusion by targeting miR-873," Cell Death and Differentiation, vol. 23, no. 8, pp. 1394-1405, 2016.

[21] D. Zheng, M. Huo, B. Li et al., "The role of exosomes and exosomal microRNA in cardiovascular disease," Frontiers in Cell and Development Biology, vol. 8, 2021.

[22] Y. Wang, L. Zhang, Y. Li et al., "Exosomes/microvesicles from induced pluripotent stem cells deliver cardioprotective miRNAs and prevent cardiomyocyte apoptosis in the ischemic myocardium," International Journal of Cardiology, vol. 192, pp. 61-69, 2015.

[23] W. Minghua, G. Zhijian, H. Chahua et al., "Plasma exosomes induced by remote ischaemic preconditioning attenuate myocardial ischaemia/reperfusion injury by transferring miR-24," Cell Death \& Disease, vol. 9, no. 3, p. 320, 2018.

[24] T. Sun, W. Ding, T. Xu et al., "Parkin regulates programmed necrosis and myocardial ischemia/reperfusion injury by targeting cyclophilin-D," Antioxidants \& Redox Signaling, vol. 31, no. 16, pp. 1177-1193, 2019.

[25] H. Tan, J. Qi, B. Y. Fan, J. Zhang, F. F. Su, and H. T. Wang, "MicroRNA-24-3p attenuates myocardial ischemia/reperfusion injury by suppressing RIPK1 expression in mice," Cellular Physiology and Biochemistry, vol. 51, no. 1, pp. 46-62, 2018.

[26] X. Lv, P. Lu, Y. Hu, and T. Xu, "miR-346 inhibited apoptosis against myocardial ischemia-reperfusion injury via targeting Bax in rats," Drug Design, Development and Therapy, vol. Volume 14, pp. 895-905, 2020.

[27] F. Wang and H. Tong, "Precondition of sevoflurane upregulates TIMP3 expression to alleviate myocardial ischemia/reperfusion injury," Perfusion, vol. 36, no. 7, pp. 717-723, 2021.

[28] B. P. Purcell, S. C. Barlow, P. E. Perreault et al., "Delivery of a matrix metalloproteinase-responsive hydrogel releasing TIMP-3 after myocardial infarction: effects on left ventricular remodeling," American Journal of Physiology. Heart and Circulatory Physiology, vol. 315, no. 4, pp. H814-H825, 2018.

[29] H. Liu, X. Jing, A. Dong, B. Bai, and H. Wang, “Overexpression of TIMP3 protects against cardiac ischemia/reperfusion injury by inhibiting myocardial apoptosis through ROS/Mapks pathway," Cellular Physiology and Biochemistry, vol. 44, no. 3, pp. 1011-1023, 2017.

[30] T. Fujii, S. Duarte, E. Lee, B. Ke, R. W. Busuttil, and A. J. Coito, "Tissue inhibitor of metalloproteinase 3 deficiency disrupts the hepatocyte E-cadherin/ $\beta$-catenin complex and induces cell death in liver ischemia/reperfusion injury," Liver Transplantation, vol. 26, no. 1, pp. 113-126, 2020. 